\title{
Optimización del Proceso de Adsorción de Plomo con Quitosano Modificado para ser Utilizado en el Tratamiento de Aguas
}

\author{
C. GISELA DÁVILA MOLINA ${ }^{*}$; PABLO BONILLA \\ a Laboratorio de Control de Calidad Nefrocontrol S.A., Los Arupos E 1-129 y Av. 10 de Agosto, Quito-Ecuador \\ b Laboratorio de Coloideoquímica, Facultad de Ciencias Químicas, Universidad Central del Ecuador \\ *Correspondencia: gise_dm5@hotmail.com \\ Recibido: 7 diciembre 2010 / Aceptado: 18 abril 2011
}

\section{Resumen}

En este estudio, el quitosano fue extraído y modificado a partir de caparazones de camarón Penaeus Vannamei con el cual se prepararon dos materiales adsorbentes: quitosano-glutaraldehído (QGD) y quitosano-nitrato férrico (QFe). En la primera etapa experimental fue seleccionado el material adsorbente (QGD) que presenta un mayor porcentaje de adsorción. En las etapas experimentales posteriores son analizadas las influencias de factores como el pH, la dosificación del adsorbente, velocidad de agitación, temperatura y tiempo de contacto en la adsorción de plomo en QGD; estableciéndose en la optimización que son determinantes: el pH, la dosificación del adsorbente y el tiempo de contacto en un rango de 6,75-7,00; 0,35-0,50 g y 151-175 min respectivamente. Teniéndose una eficiencia en remoción de plomo con QGD de 98,40\% y encontrándose a partir del estudio isotérmico que la capacidad adsorbente de este material es de $282,61 \mathrm{mgPb}^{2+} / \mathrm{gQGD}$

Palabras clave: quitina, quitosano, quitosano-glutaraldehído, quitosano-hierro, adsorción, plomo.

\section{Optimization of the Lead Adsorption Process with Modified Chitosan for being used in the Treatment of Water}

\section{Abstract}

In this study, chitosan extracted from Penaeus Vannamei shrimp was modified to prepare two adsorbent materials: chitosan-glutaralaldehyde (QGD) and chitosan-ferric nitrate (QFe). Effects of the pH, dosage of adsorbent, stirring speed, temperature and contact time were evaluated. The best lead adsorption was found to occur onto QGD at $\mathrm{pH} 6,75-7,00$; dosage of adsorbent $0,35-0,50 \mathrm{~g}$ and time of contact 151-175min. The efficiency of lead remotion with $Q G D$ was $98,40 \%$ and the isothermal study of adsorptive capacity showed an absorption of $282,61 \mathrm{mg}$ of $\mathrm{Pb}^{2+}$ per $\mathrm{g}$ of $\mathrm{QGD}$.

Key words: chitosan, chitin, chitosan-glutaraldehyde, chitosan-iron, adsorption, lead.

\section{Introducción}

La contaminación de plomo proviene principalmente de fuentes como las soldaduras de tuberías, pilas y baterías, aparatos electrónicos, objetos de vidrio y cerámica, también en pinturas, en plásticos como el PVC que es utilizado en tuberías de distribución de agua potable, en vertidos industriales y labores mineras. Generándose así, aguas con altas concentraciones de plomo que son perjudiciales para el medio ambiente, puesto que el plomo es causante de graves daños en los seres vivos y es capaz de permanecer largo 
tiempo en el entorno, circulando o acumulándose en sedimentos y organismos vivos, de este modo es como se incorpora a las cadenas alimentarias [1].

Consecuentemente se vuelve necesario encontrar tecnologías no convencionales que eviten la entrada de plomo en medios acuáticos; además, concientizar a las industrias sobre la reducción de la concentración de plomo hasta niveles que no generen problemas de toxicidad.

Entre las técnicas convencionales usadas en la remoción de metales pesados se tiene: la precipitación química, intercambio iónico, tecnología de membranas y tratamiento electroquímico [2]; sin embargo, en estos días ha tomado gran importancia la biosorción ya que es una "tecnología limpia" que puede ser usada en los procesos de remediación ambiental, para recuperación de metales y descontaminación de aguas residuales contaminadas con iones de metales pesados. Se basa en la utilización de desechos orgánicos que evitan problemas de contaminación porque pueden regenerarse y no son costosos, ya que en general son procedentes de residuos de industrias alimenticias, como es el caso de este estudio, en el que fueron utilizados desechos de la industria camaronera, de donde es posible obtener quitosano como material adsorbente, cuyas propiedades acomplejantes con iones metálicos, en general, muestran alta eficiencia en la remoción de mercurio, cromo, cobre, zinc, entre otros; motivo por el cual fue considerado en este estudio, pretendiéndose la optimización del proceso de adsorción de plomo y al mismo tiempo la reutilización de los residuos provenientes de la industria camaronera, que en nuestro país es uno de los principales productos de exportación y que aún no se encuentra ampliamente difundida.

\section{Materiales y Métodos}

\subsection{Materiales}

Se utilizaron caparazones de camarón Penaeus vannamei del mercado América. Para la extracción de quitina se utilizaron soluciones de ácido clorhídrico $1 \mathrm{~N}$ e hidróxido de sodio $1 \mathrm{~N}$. La desacetilación termoalcalina de quitina para su conversión a quitosano fue realizada con hidróxido de potasio $70 \%$. En la síntesis de los materiales adsorbentes provenientes de quitosano fue utilizado ácido acético $2 \%$, glutaraldehído $2,5 \%$, nitrato férrico nonahidratado $0,01 \mathrm{~N}$. Para la optimización del proceso de adsorción se utilizó una solución estándar de 1.000 ppm de plomo.

\subsection{Equipos}

Se utilizó un espectrómetro de absorción atómica Thermo Electron S Series AA Spectrometer para la determinación de plomo en las soluciones acuosas ensayadas.

\subsection{Diseño de experimentos}

El diseño de experimentos y el análisis estadístico en este estudio fue llevado a cabo usando el software estadístico Minitab 15 [6]. El diseño de experimentos comprendió tres etapas. Cribado, en el cual se determinó el adsorbente de mayor eficiencia, comparándolo con zeolita (clinoptilolita) que tiene buenas propiedades de adsorción para plomo, como criterio de selección; también se analizó la concentración de plomo con la cual se trabajaría en las siguientes etapas, tomándose en cuenta el bloqueo de las otras variables, teniéndose un diseño de dos factores $y$ tres niveles que al ser replicado a un total de 18 experimentos. Fue utilizado un diseño de Plackett-Burman en el cual fueron analizados como factores: la dosificación del adsorbente, el pH, el tiempo de contacto, la velocidad de agitación y la temperatura [4], determinado así qué factores eran predominantes en la adsorción de plomo, incluyendo dos réplicas, teniéndose 24 experimentos. Finalmente, se llevó a cabo un diseño de optimización Box-Behnken, en el cual fueron analizados el $\mathrm{pH}$, la dosificación del adsorbente y el tiempo de contacto.

\subsection{Procedimiento experimental}

\subsubsection{Extracción de quitina [3]}

Se realizó la desmineralización con $\mathrm{HCl} 1 \mathrm{~N}$ durante tres horas, considerando que debe existir una relación 1:10 entre la cantidad de caparazones y el volumen de ácido, se neutralizó con abundante agua destilada. Posteriormente, se realizó la desproteinización utilizando $\mathrm{NaOH} 1 \mathrm{~N}$ durante tres horas, considerando una relación $1: 15$, se neutralizó con abundante agua destilada. Se secó la quitina obtenida a una temperatura de $60^{\circ} \mathrm{C}$. 


\subsubsection{Obtención de quitosano}

La desacetilación fuertemente alcalina se realizó con $\mathrm{KOH} 2 \mathrm{~N}$ durante cuatro horas a una temperatura de $105^{\circ} \mathrm{C}$ considerando una relación de 1:15 entre quitina y volumen de $\mathrm{KOH} 2 \mathrm{~N}$.

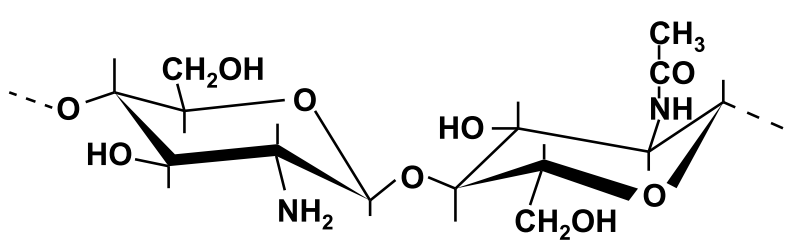

Figura 1. Unidad repetitiva de quitosano

\subsubsection{Síntesis de materiales adsorbentes derivados de} quitosano

\subsubsection{Reticulación de quitosano con glutaraldehído [5]}

Se preparó una solución al $2 \%$ de quitosano en $\mathrm{CH}_{3} \mathrm{COOH}$ al $2 \%$ mediante agitación hasta formar una solución viscosa, esta es dosificada sobre una solución de $\mathrm{KOH} 2 \mathrm{~N}$, formándose así las esferas de quitosano las cuales después de ser lavadas con agua destilada hasta neutralización fueron colocadas en glutaraldehído 2,5\% agitándose durante 6 horas.

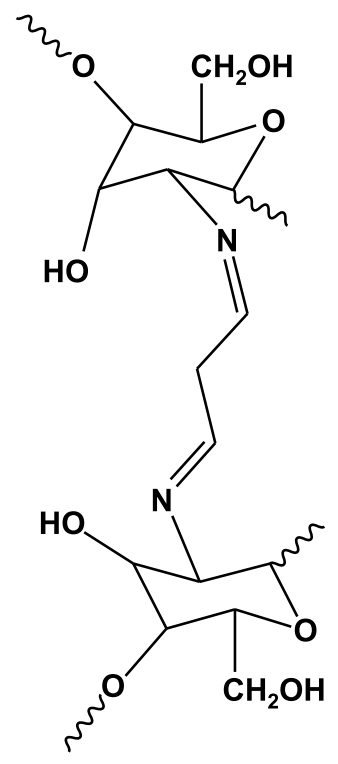

Figura 2. Quitosano-Glutaraldehído

\subsubsection{Quitosano-nitrato férrico $[5,7]$}

Se preparó una solución al $2 \%$ de quitosano en nitrato férrico nonahidratado $0,01 \mathrm{~N}$ acidificado con ácido acético, obteniéndose una solución viscosa, la cual fue dosificada sobre una solución de hidróxido de potasio $2 \mathrm{~N}$, lavándose posteriormente con agua destilada hasta neutralización.

\subsubsection{Optimización de la adsorción de plomo en quitosano}

La optimización del proceso de adsorción de plomo sobre quitosano fue estructurada en las etapas como se muestra en la Tabla 1.

Tabla 1. Estructuración de factores y niveles en el diseño de experimentos propuestos

\begin{tabular}{|c|c|c|c|}
\hline $\begin{array}{l}\text { Tipo de } \\
\text { diseño }\end{array}$ & Factores & Niveles & $\begin{array}{c}\text { Parámetro de } \\
\text { medición }\end{array}$ \\
\hline \multirow{2}{*}{ Cribado } & $\begin{array}{c}\text { Tipo de } \\
\text { adsorbente }\end{array}$ & $\begin{array}{l}\text { QGD, QFe, } \\
\text { Zeolita }\end{array}$ & \multirow{10}{*}{$\begin{array}{l}\text { \% Remoción } \\
\text { de plomo }\end{array}$} \\
\hline & $\begin{array}{c}\text { Concentración } \\
\text { óptima }\end{array}$ & 10,20 y 30 ppm & \\
\hline \multirow{5}{*}{$\begin{array}{l}\text { Plackett- } \\
\text { Burman }\end{array}$} & $\mathrm{pH}$ & $5,0-7,0$ & \\
\hline & $\begin{array}{c}\text { Dosificación } \\
\text { de adsorbente }\end{array}$ & $0,10-0,30 \mathrm{~g}$ & \\
\hline & $\begin{array}{l}\text { Tiempo de } \\
\text { contacto }\end{array}$ & $75-150 \mathrm{~min}$ & \\
\hline & Temperatura & $25-40^{\circ} \mathrm{C}$ & \\
\hline & $\begin{array}{l}\text { Velocidad de } \\
\text { agitación }\end{array}$ & $100-200$ rpm & \\
\hline \multirow{3}{*}{ Box-Behnken } & $\begin{array}{l}\text { Dosificación } \\
\text { adsorbente }\end{array}$ & $\begin{array}{c}0,30 \mathrm{~g} ; 0,45 \mathrm{~g} \\
0,60 \mathrm{~g}\end{array}$ & \\
\hline & $\mathrm{pH}$ & 6,$50 ; 6,75 ; 7,00$ & \\
\hline & $\begin{array}{l}\text { Tiempo de } \\
\text { contacto }\end{array}$ & $\begin{array}{c}150,165 \text { y } 180 \\
\min \end{array}$ & \\
\hline
\end{tabular}

\subsubsection{Determinación de la capacidad de adsorción del material adsorbente y estudio cinético}

Se determinó la capacidad de adsorción del material utilizando los modelos planteados por Langmuir y Freundlich, preparando una serie de soluciones de concentraciones de 10 a 100 ppm de plomo, las cuales fueron sometidas a las condiciones establecidas como óptimas para la adsorción. 


\section{Resultados y Discusión}

\subsection{Extracción de quitina y obtención de quitosano}

De acuerdo a la metodología utilizada para la extracción de quitina se tuvo un rendimiento del $7,08 \%$. Posteriormente, se realizó la desacetilación termoalcalina de quitina, teniéndose un rendimiento del $68,18 \%$; las propiedades del material obtenido se muestran en la Tabla 2.
Tabla 2. Ensayos complementarios para caracterización de quitosano

\begin{tabular}{cc}
\hline Método de caracterización & Resultados obtenidos \\
\hline Grado de desacetilación & $83,34 \%$ \\
Pérdida por desecación & $10,26 \%$ \\
Sólidos insolubles & $0,62 \%$ \\
Cenizas & $0,88 \%$ \\
Contenido de nitrógeno & $6,74 \%$ \\
\hline
\end{tabular}

\subsection{Síntesis de materiales adsorbentes derivados de quitosano}

Las propiedades de los materiales adsorbentes, objetos de este estudio, se muestran en la Tabla 3.

Tabla 3. Características materiales adsorbentes derivados de quitosano

\begin{tabular}{ccc}
\hline Material adsorbente & Densidad aparente $\mathbf{( g / \mathbf { c m } ^ { 3 } )}$ & \%Humedad \\
\hline Quitosano-Glutaraldehído & 0,8421 & 83,98 \\
Quitosano-Nitrato Férrico & 0,8373 & 91,31 \\
\hline
\end{tabular}

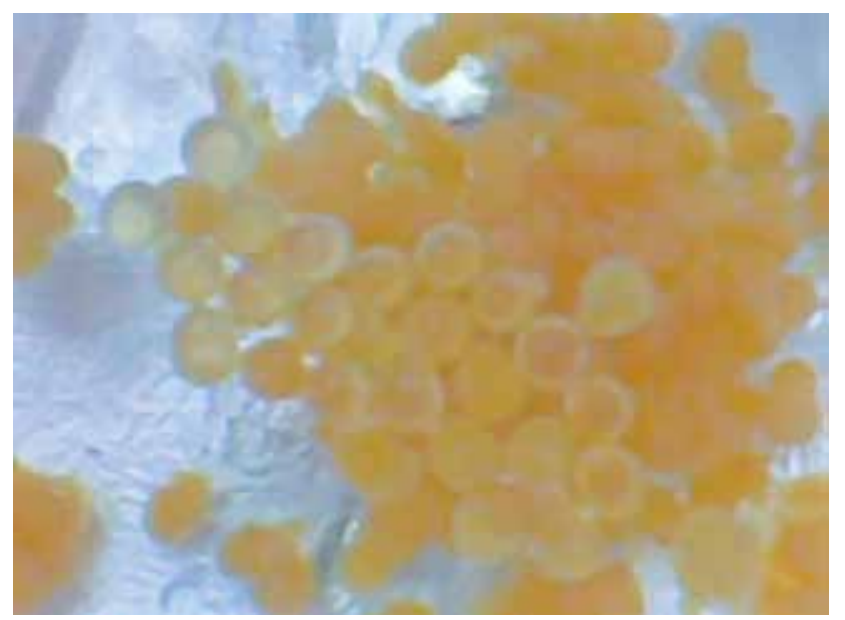

Figura 3. Quitosano-Nitrato Férrico

\subsection{Optimización del proceso de adsorción de plomo}

\subsubsection{Cribado}

De acuerdo al análisis de varianza realizada, se tuvo que el factor determinante es el tipo de adsorbente y que existe una interacción importante entre

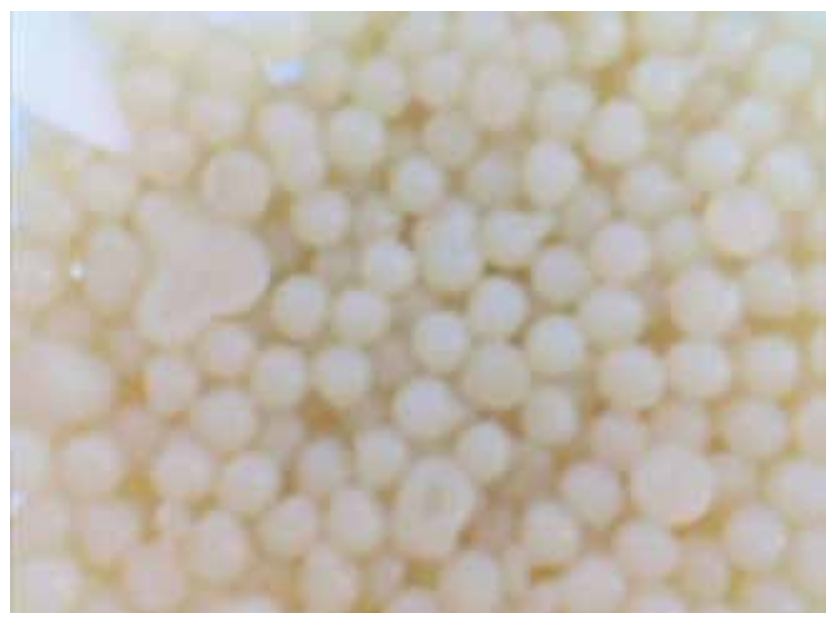

Figura 4. Quitosano-Glutaraldehído

tipo de adsorbente y concentración. El tipo de adsorbente es seleccionado en función de su comportamiento similar al de la zeolita, es decir, el que presenta una mejor respuesta en el porcentaje de remoción de plomo, siendo quitosano-glutaraldehído frente a una concentración de 10 ppm de plomo; esta es la mejor combinación entre los factores analizados. 


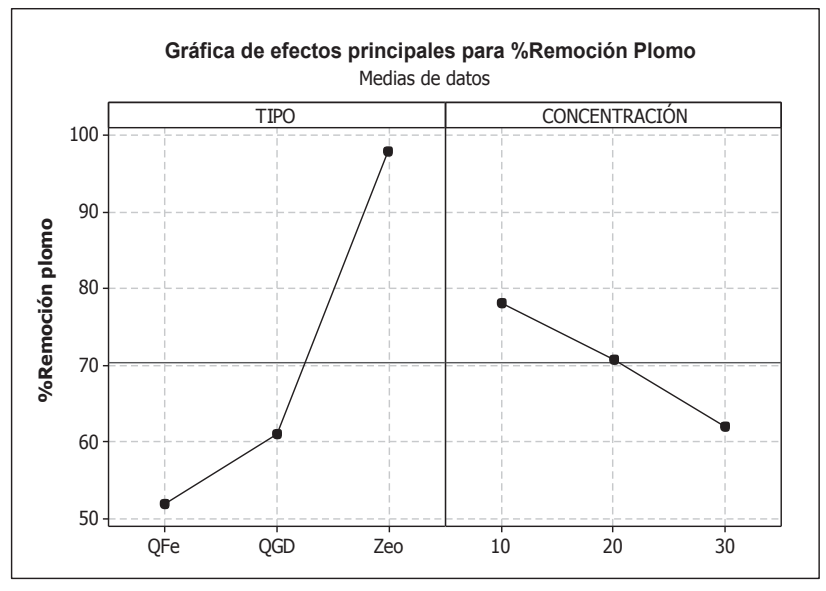

Figura 5. Gráfico de efectos principales-cribado

\subsubsection{Plackett-Burman}

Se estableció que el efecto principal de este diseño es la dosificación del adsorbente; sin embargo, por medio del análisis de varianza se determinó que este factor no puede explicar por sí solo el modelo de adsorción, siendo considerados como importantes también los efectos de $\mathrm{pH}$ y tiempo de contacto.

\subsubsection{Box-Benhken}

Por medio del software estadístico Minitab 15 se obtuvieron las siguientes gráficas de superficies que muestran las correspondientes interacciones por pares de los factores analizados:

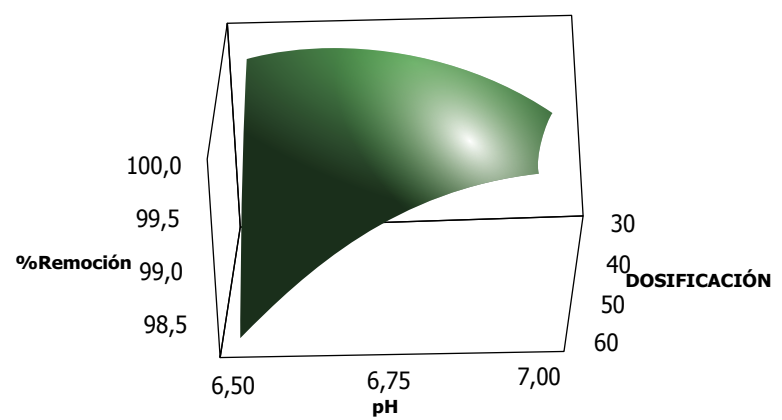

Figura 6. Superficie \% Remoción $\mathrm{Pb}^{2+}$ vs. $\mathrm{pH}$. Dosificación

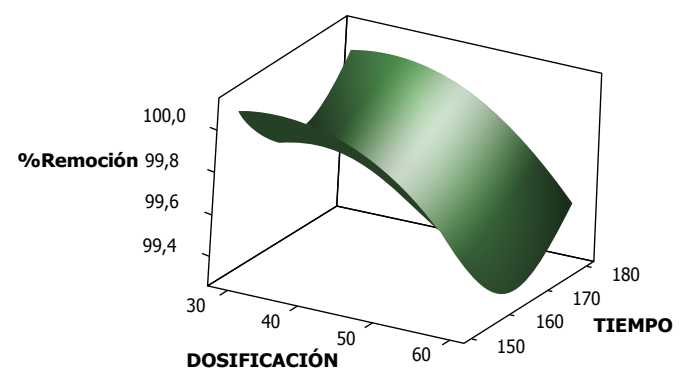

Figura 7. Superficie \% Remoción $\mathrm{Pb}^{2+}$ vs. tiempo

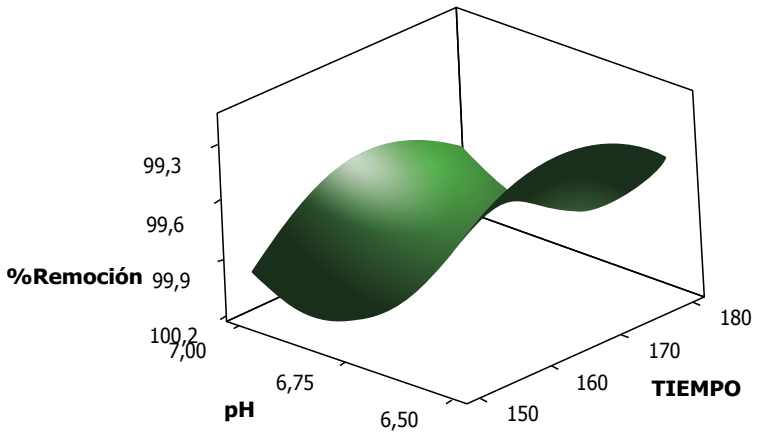

Figura 8. Superficie \% Remoción $\mathrm{Pb}^{2+}$ vs. tiempo. $\mathrm{pH}$

Teniéndose áreas óptimas de adsorción en $\mathrm{pH}$ de 6,75-7,00; dosificación de 0,35g-0,50 g y un tiempo de contacto de $151<t<175 \mathrm{~min}$.

Una de las herramientas del software estadístico Minitab 15 es el optimizador de respuesta, con el cual se obtuvo que los óptimos teóricos para el fenómeno de adsorción se tenían con una dosificación de 0,3279 g; un $\mathrm{pH}$ de 6,72 y un tiempo de 153,26 min; bajo estos óptimos teóricos fueron realizados ensayos; teniéndose que el porcentaje de adsorción de plomo es de $98,40 \%$ con lo cual se tiene un error versus optimización teórica $(100 \%)$ de 1,598\%.

\subsection{Determinación de la capacidad de adsorción máxima y estudio cinético de adsorción}

Se determinó que la adsorción de plomo en quitosanoglutaraldehído se rige bajo el modelo de Freundlich, asumiéndose con esto que se da la adsorción de moléculas formando una monocapa de superficie heterogénea, encontrándose que la capacidad de adsorción máxima es de 282,61 mg Pb²+/gQGD

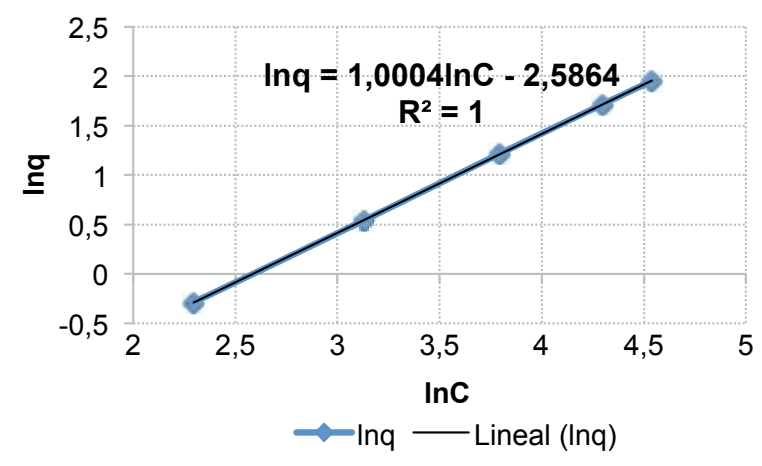

Figura 9. Isoterma de Freundlich para adsorción de plomo en $Q G D$ 
El estudio cinético de la adsorción de plomo en quitosano-glutaraldehído, se ajusta al modelo cinético de pseudo segundo orden; esto supone entonces que el plomo es adsorbido en dos sitios activos, dados por el grupo imino y el grupo hidroxilo, presentes en la molécula; además, se denota que se da la adsorción hasta el tiempo estimado como óptimo.

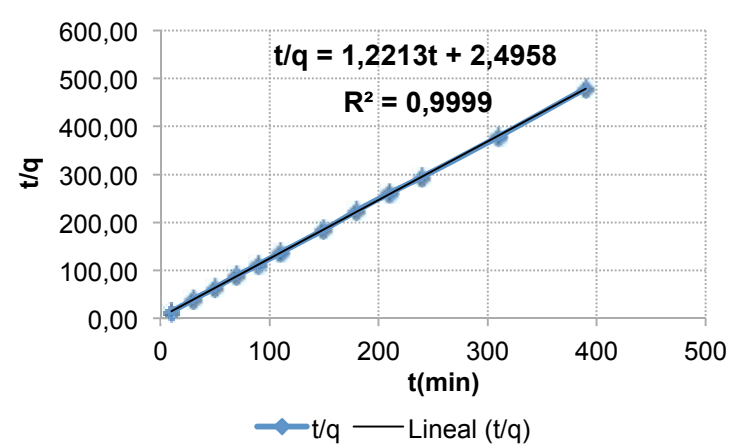

Figura 10. Modelo cinético de pseudo segundo orden para adsorción de plomo en $Q G D$

\section{Conclusiones}

El porcentaje de remoción de plomo utilizando quitosano-glutaraldehído es de $98,40 \%$, siendo entonces un excelente material adsorbente para la eliminación de plomo en soluciones acuosas, pudiendo ser una alternativa eficiente y de paso que propone la reutilización de desechos de la industria camaronera en Ecuador. Además de eso, las condiciones de adsorción para la eliminación de plomo han sido optimizadas con éxito. Las condiciones óptimas encontradas son de 0,3279 $\mathrm{g}$ de quitosano-glutaraldehído; $\mathrm{pH}$ de 6,72 y tiempo de contacto de 153,26 min; bajo estas condiciones óptimas se obtuvo que la capacidad de adsorción máxima de plomo en QGD es de $282,61 \mathrm{mgPb}^{2+} / \mathrm{gQGD}$.

\section{Agradecimientos}

El presente estudio pudo ser realizado en las instalaciones de Nefrocontrol S.A.; agradezco el apoyo y ayuda brindados por la Dra. Lourdes Pazos G. De igual manera, mi agradecimiento al lng. Daniel Pazos, pilar fundamental durante el desarrollo de este trabajo, por su amistad, guía y asesoría incondicional.

Asimismo, deseo agradecer la asistencia proporcionada por el Dr. Ronny Flores, profesor de la Facultad de Ciencias Químicas de la Universidad Central del Ecuador.

\section{Referencias}

1. Aprovechame-Ministerio de Educación y Cultura de España. (s.f.). Reutilización de residuos de rapa para la eliminación de metales tóxicos en efluentes líquidos. (Scirus) Recuperado el 06 de marzo de 2009, de Aprovechamiento de residuos vegetales procedentes de procesos industriales para la concentración y separación de iones metálicos-Aprovechame: www.scirus.com

2. Volesky, B. Detoxification of metal-bearing efluents: biosorption for the next century. Hydrometallurgy. No.59, (2001), p. 203-216.

3. Peniche Cavas, C. A. (2006). Estudios sobre Quitina y Quitosano. La Habana.

4. Kabbashi, N., Nour, A. H., Muyibi, S., \& Qudsieh, I. Y. (2009). Development of a wastewater treatment system for chromium removal using chitosan.

5. Díaz de Apodaca, E., Villarán Velasco, M. C., Río Pérez, F. R., \& Ibarrera, L. L. (2007). Utilizado de adsorbentes basados en quitosano y alginato sódico para la eliminación de iones metálicos: $\mathrm{Cu}^{2+}, \mathrm{Pb}^{2+}, \mathrm{Cr} 3+, \mathrm{Co}^{2+}$.

6. Minitab Inc., "Minitab registrado", versión 15.120.0, Microsoft Windows XP, 2006

7. Vassireddy, D. (2005). Arsenic adsorption onto iron-chitosan composite from drinkin water. Missouri: Universidad Missouri-Columbia 